\title{
An acoustic backscattering technique for the detection of transient cavitation produced by microsecond pulses of ultrasound
}

Ronald A. Roy, Sameer I. Madanshetty, and Robert E. Apfel

Citation: The Journal of the Acoustical Society of America 87, 2451 (1990); doi: 10.1121/1.399091

View online: https://doi.org/10.1121/1.399091

View Table of Contents: https://asa.scitation.org/toc/jas/87/6

Published by the Acoustical Society of America

\section{ARTICLES YOU MAY BE INTERESTED IN}

Acoustic microcavitation: Its active and passive acoustic detection

The Journal of the Acoustical Society of America 90, 1515 (1991); https://doi.org/10.1121/1.401891

Ultrasonic cavitation monitoring by acoustic noise power measurement

The Journal of the Acoustical Society of America 108, 2012 (2000); https://doi.org/10.1121/1.1312360

Thresholds for transient cavitation produced by pulsed ultrasound in a controlled nuclei environment

The Journal of the Acoustical Society of America 88, 2059 (1990); https://doi.org/10.1121/1.400102

Passive cavitation mapping for localization and tracking of bubble dynamics

The Journal of the Acoustical Society of America 128, EL175 (2010); https://doi.org/10.1121/1.3467491

An analysis of the acoustic cavitation noise spectrum: The role of periodic shock waves

The Journal of the Acoustical Society of America 140, 2494 (2016); https://doi.org/10.1121/1.4964633

The Role of Impurities in Cavitation-Threshold Determination

The Journal of the Acoustical Society of America 48, 1179 (1970); https://doi.org/10.1121/1.1912258 


\title{
An acoustic backscattering technique for the detection of transient cavitation produced by microsecond pulses of ultrasound
}

\author{
Ronald A. Roy, ${ }^{\text {a) }}$ Sameer I. Madanshetty, ${ }^{\text {b) }}$ and RobertE. Apfel \\ Department of Mechanical Engineering, Yale University, Box 2159, Yale Station, New Haven, \\ Connecticut 06520
}

(Received 28 September 1989; accepted for publication 16 January 1990)

\begin{abstract}
An acoustic backscattering technique for detecting transient cavitation produced by $10-\mu \mathrm{s}$-long pulses of $757-\mathrm{kHz}$ ultrasound is described. The system employs $10-\mu \mathrm{s}-$ long, $30-\mathrm{MHz}$ center frequency tone bursts that scatter from cavitation microbubbles. Experiments were performed with suspensions of hydrophobic polystyrene spheres in ultraclean water. Transient cavitation threshold pressures measured with the active cavitation detector (ACD) were always less than or equal to those measured using a passive acoustic detection scheme. The measured cavitation thresholds decreased with increasing dissolved gas content and increasing suspended particle concentration. Results also show that ultrasonic irradiation of the polystyrene sphere suspensions by the ACD lowered the threshold pressure measured with the passive detector. A possible mechanism through which suspensions of hydrophobic particles might nucleate bubbles is presented.
\end{abstract}

PACS numbers: 43.30.Ft, 43.30.Gv, 43.25.Yw, 43.35.Ei

\section{INTRODUCTION}

Since its introduction, pulsed ultrasound has established itself as an effective diagnostic tool in clinical radiology. This acceptance led to a pattern of frequent and widespread use that has served to fuel a concern over the potential health risks associated with ultrasonic irradiation. One of the principal routes to ultrasonically induced bioeffects is by way of acoustic cavitation. Up until a few years ago, it was generally felt that the short pulses of high-frequency ultrasound that are characteristic of diagnostic scanners (the pulse center frequency, duration, and repetition frequency are typically $5 \mathrm{MHz}, 2$ cycles, and $1 \mathrm{kHz}$, respectively) could not induce cavitation due to the low time-average power levels and the brief insonofication intervals. However, Apfel ${ }^{1}$ and Flynn ${ }^{2}$ have independently predicted that transient cavitation can result by insonifying a free microbubble with a single cycle of megahertz-frequency ultrasound. Most recently, Holland and Apfel ${ }^{3}$ calculated the minimum acoustic stress required to produce transient cavitation (i.e., the cavitation threshold) given 1-MHz, single-cycle irradiation in water. Their predicted threshold pressure amplitudes ranged as low as 2.5 bars, which stands in stark contrast with the spatial-peak-temporal-peak (SPTP) pressures encountered in clinical ultrasonic scanners, some of which exceed 40 bars.

Theoretical predictions not withstanding, there is relatively little experimental evidence to support the contention that diagnostic ultrasound can produce cavitation. This may be due in part to the fact that cavitation is by nature a dis-

\footnotetext{
") Current address: National Center for Physical Acoustics, P.O. Box 847, University, MS 38677.

b) Current address: Division of Applied Sciences, Harvard University, 12Gf Pierce Hall, 29 Oxford Street, Cambridge, MA 02138.
}

crete phenomenon that is both spatially and temporally localized. However, cavitation does manifest itself in a number of observable processes such as bubble formation, free radical generation, and light production (see Ref. 4 for a comprehensive discussion of acoustic cavitation inception and bubble dynamics). The detectability of cavitation activity is dependent upon the mechanism employed as an indicator. Keying on chemiluminescence light production, Fowlkes and Crum $^{5}$ measured thresholds as low as 8 bars in a relatively dirty and gassy luminol solution irradiated with single-cycle pulses of low-duty-cycle ultrasound. Although well in excess of theoretical predictions, their pressures were lower than those produced by most clinical devices. Atchley et al. ${ }^{6}$ employed an acoustic transducer to listen passively for sound scattered by cavitation microbubbles. This technique, which we term passive cavitation detection, yielded threshold values lower than those measured via light production, yet higher than theoretical predictions based on free-bubble cavitation models.

Both of the above detection schemes exhibited a reduction in the measured threshold with increasing pulse duration for a constant pulse-repetition frequency (PRF). However, theory predicts that if transient cavitation were to occur, it would commence in the first acoustic cycle (for a free-bubble nuclei). Therefore, the pulse duration should not be critical in determining the cavitation threshold. Acoustic cavitation is an evolutionary process, generally starting with the nucleation of a single bubble and often culminating with the formation of a bubble cloud. Depending on the detector sensitivity, a detectable amount of generated light or scattered sound might require the formation of a bubble cloud as opposed to the generation of an individual bubble. Limitations in the sensitivity of cavitation detectors can be a significant drawback in systems employing very 
short pulses and low duty cycles in media that is either acoustically lossy or affords limited optical access.

In this article we introduce a high-frequency, narrowband, acoustic pulse-echo technique for transient microcavitation detection. When suitably implemented, this active cavitation detector (ACD) affords sufficient sensitivity and temporal resolution to noninvasively detect the presence of a single $1-\mu \mathrm{m}$ bubble that lasts only a microsecond. In addition to the ACD, we employ a passive acoustic detector similar to the one described in Ref. 6. Multiple detection allows for direct comparison of the two techniques. Measurements are performed in chemically and physically clean, degassed water seeded with polystyrene latex microspheres. This choice of working medium, although clinically irrelevant, facilitates the precise control of liquid properties such as viscosity, surface tension, dissolved gas concentration, and cavitation nuclei concentration. By reducing the number of unknown liquid parameters, we are better equipped to interpret unambiguously threshold measurements in light of existing bubble theories.

\section{EXPERIMENTAL APPARATUS}

The apparatus is composed of a test chamber, a fluidhandling apparatus, and three instrumentation systems. The cavitation test chamber allows for ultrasonic irradiation of a test sample while monitoring for cavitation activity using simultaneous active and passive detectors. A closed, recirculating filtration network cleans and degasses the water, flushes the test chamber, and facilities the uniform suspension of cavitation nuclei. The three instrumentation systems, diagrammed in Fig. 1, consist of a $757-\mathrm{kHz}$ high-intensity acoustic tone-burst generator for cavitation production, a 30-MHz pulse-echo active cavitation detector, and a passive cavitation detector. In this article, we shall refer to the cavitation-production and cavitation-detection fields as the primary and detector fields, respectively.

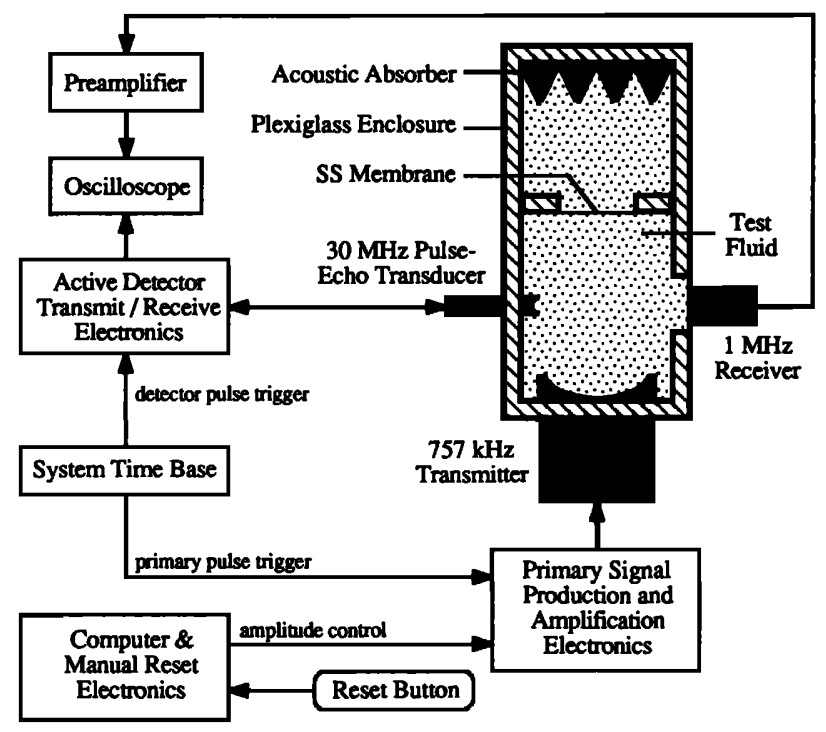

FIG. 1. Block diagram of the experimental apparatus.

\section{A. Cavitation test tank and fluid-handling system}

A top view of the test tank is given in Fig. 1. This watertight Plexiglas enclosure consists of a 7.5-cm-wide, $15.5-\mathrm{cm}$ high, and $26.5-\mathrm{cm}$-long box partitioned into two sections. The 1.5- $\ell$-capacity forward section serves as the cavitation test cell. To inhibit standing-wave generation, we employ an acoustic absorber housed in the rearward section. A 9- $\mu \mathrm{m}$ thick, acoustically transparent, stainless-steel membrane serves to isolate the contents of the two sections. The primary-field transducer, which irradiates the cavitation cell along the elongated axis of the enclosure, is mounted on a $X$ $Y-Z$ translation stage. This facilitates the confocal orientation of the primary- and detector-field transducers, the latter being rigidly mounted in the side wall of the enclosure. Passive detection is achieved by affixing (with coupling gel) a nonfocused transducer to the outside of the cell at a point directly.opposite the active detector.

Test samples are processed using the closed-flow system diagrammed in Fig. 2. A $20-\ell$ Plexiglas reservoir feeds a magnetic-drive gear pump that forces the water through inline de-ionization, organic removal, and $0.2-\mu \mathrm{m}$ microfiltration stages. The flow then enters a lower corner of the cavitation cell, exits through a port in the cover, and is deposited in the reservoir. This recirculating system was designed to attain flow rates of up to $5 \mathrm{\ell} / \mathrm{min}$ and thus thoroughly flushes the cavitation cell, removing almost all dirt and impurities from the cell walls. Degassing is accomplished by using the vacuum pump and bleed valve to control the air pressure in the reservoir while simultaneously recirculating the water. This action results in the liberation of dissolved gas until the partial pressure of the dissolved air becomes equal to the air pressure above the water's surface (with a small correction for vapor pressure). We then turn off the circulation pump, gradually release the vacuum, draw off a sample from the reservoir, and measure the air content with a dissolved oxygen mreter. Repeated measurements suggest that the air concentration in the test sample changes by no more than $3 \%$ over the duration of an experiment.

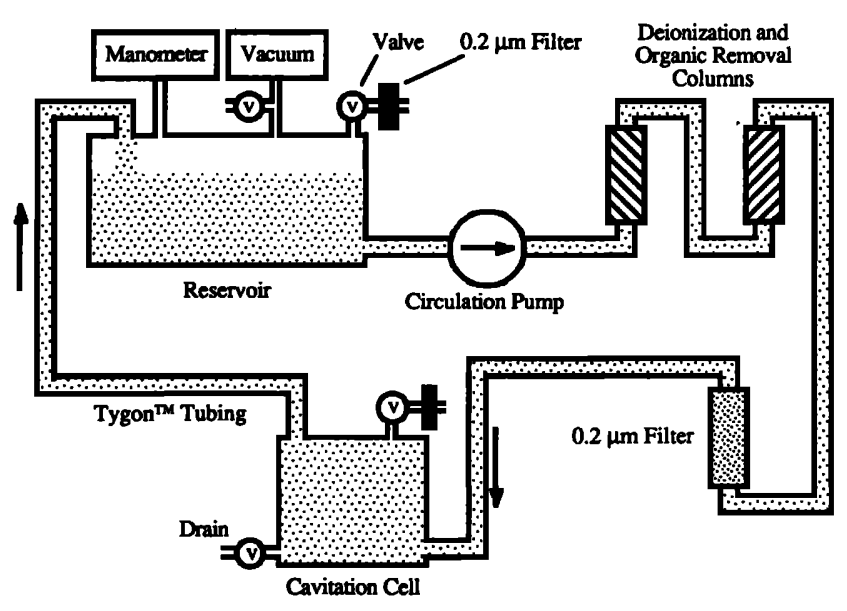

FIG. 2. Closed-flow circulation system for cleansing and degassing the sample liquid. 


\section{B. Primary-field production system}

Figure 3 is a block diagram of the instrumentation used to generate the high-intensity ultrasonic pulses with which we produce cavitation. We obtain electrical tone bursts by gating a 757-kHz continuous-wave signal with a rectangular pulse produced by a gate-and-delay generator. The width of the gating pulse dictates the duration of the tone burst. (See Refs. 7 and 8 for a more detailed description of this and other instrumentation techniques employed in this work.) The burst is then routed through a voltage-controlled attenuator and a power amplifier that drives the primary-field transducer. Automated control of the primary-field pressure amplitude is achieved by using the digital-to-analog output of a microcomputer to drive the voltage-controlled attenuator.

The primary-field transducer is comprised of a $3.81-\mathrm{cm}$ diam, 757-kHz PZT crystal. This undamped, spherical segment has a $5.5-\mathrm{cm}$ focal length and a $-3-\mathrm{dB}$ transverse beamwidth of about $3.5 \mathrm{~mm}$ measured along the focal plane. We calibrated the source against a Marconi PVDF membrane hydrophone using a substitution method. ${ }^{9}$ At high pressures, finite-amplitude effects lead to an asymmetry in the acoustic pulse, with the positive pressures exceeding the negative pressures. It is the negative acoustic pressure cycle that is responsible for generating the stresses that result in cavitation inception. Therefore, all of the pressure amplitudes cited in this article refer to peak negative acoustic pressures.

\section{Passive cavitation detection system}

When we irradiate the test cell with the primary field and passively listen at $90^{\circ}$, an extremely stable, low-amplitude scattered signal is detected due to multiple-path scattering from stationary surfaces within the chamber. This "background," which we monitor with a 1-MHz nonfocused receiver is shown in the top trace in Fig. 4. The production of a cavitation complex causes some of the primary field to be scattered, which results in a perturbation in the ambient background of the type shown in the bottom trace in Fig. 4. This enhanced scattering emanates from scatters located in the confocal region. (We determined this by simply inserting a spherical target into the transducer focus.) When produced by cavitation, perturbations of this form are highly transient and readily observable. Therefore, to passively de-

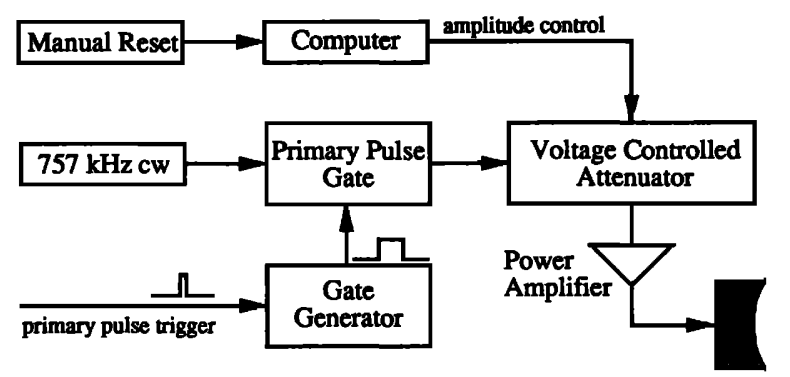

Primary Pulse Transducer

FIG. 3. Block diagram of the instrumentation for producing high-intensity ultrasonic tone bursts (i.e., the primary-field pulse) with which we generate cavitation.
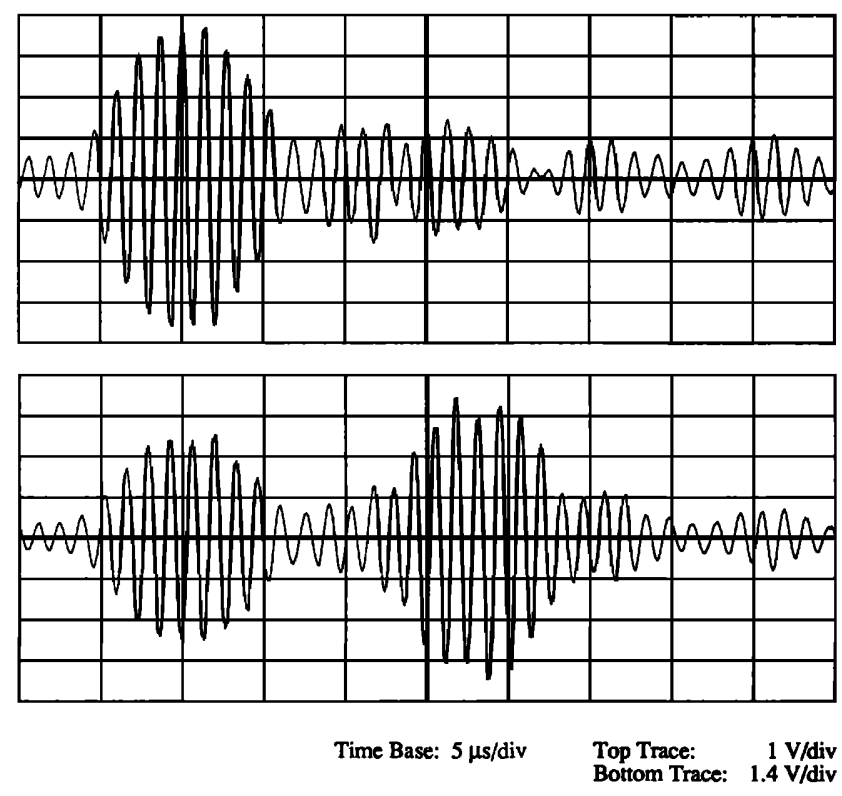

FIG. 4. The passive detector output signal in the absence and presence of cavitation (top and bottom traces, respectively). Note the difference in the vertical scales for the two traces. These waveforms were obtained with a Lecroy 9400 digital oscilloscope.

tect cavitation activity, all one has to do is monitor the preamplified output of the receiving transducer on an analog oscilloscope. There is, however, some doubt as to whether or not this system possesses sufficient sensitivity to detect the presence of a single cavitation bubble. ${ }^{9}$

\section{Active cavitation detection system}

Figure 5 is a block diagram of the instrumentation used to transmit and receive the high-frequency ultrasonic pulses with which we probe for cavitation activity. By gating a 30$\mathrm{MHz}$ continuous-wave signal, we obtain an rf tone burst that is amplified and then fed into a transmit/receive (TR) switch described in Ref. 7. In the transmit mode, the electrical burst drives a $30-\mathrm{MHz}$ narrow-band transducer that is confocally positioned relative to the primary-field transmitter. This 1.5-cm-diam, 3.0-cm-focal-length transducer has a -3-dB beamwidth (measured in the focal plane) of about $275 \mu \mathrm{m}$. In the receive mode, the backscattered signal passes through the TR switch, undergoes $60 \mathrm{~dB}$ of preamplification, and is displayed on the oscilloscope.

Transient cavitation is a fleeting phenomenon that may come and go in a few microseconds. To ensure detectability,

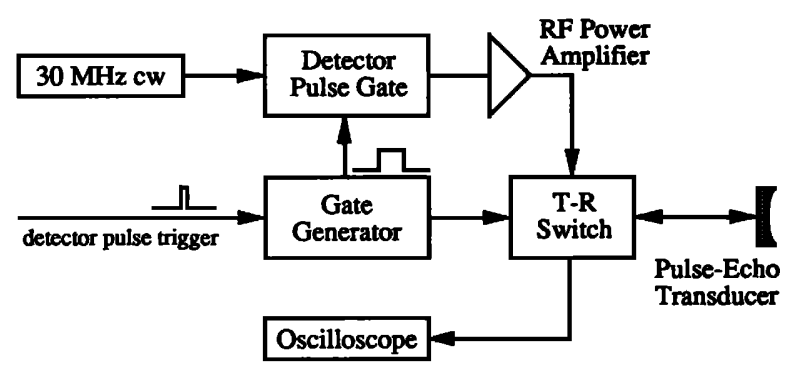

FIG. 5. Block diagram of the active cavitation detection system. 
we must make sure that the leading edges of the cavitation production and detection pulses arrive in the confocal region simultaneously. Since the focal lengths of the primary- and detector-field transducers differ by $3 \mathrm{~cm}$, we must delay the detector pulse by about $20 \mu \mathrm{s}$. The relative timing of the cavitation production and detection systems is illustrated in the timing diagram given in Fig. 6.

Shown in Fig. 7 are a series of digital oscilloscope traces that illustrate the signal generated and received by the ACD. The top trace is the primary-field electrical tone burst, and the middle trace is the output of the TR switch in the absence of cavitation. Note that a portion of the transmitted detector pulse leaks through the TR switch, which provides us with a convenient means for setting the delay between the primary and detector pulses. When cavitation does occur, it results in a backscattered signal similar to the one present in the bottom trace. Although this signal might indicate the sequential growth and collapse of three bubbles, this interpretation is complicated by the fact that the $30-\mathrm{MHz}$ carrier signal was digitized at only 100 Msamples/s; sampling error could be contributing to some of the observed structure.

Because of its high frequency and tight focusing, the ACD system employed in this work is not calibrated; thus we cannot make quantitative statements concerning the detector sensitivity. However, this system is virtually identical to a pulse-echo scattering apparatus that we designed for quantitative measurements of the backscattered echo from individual human erythrocytes. ${ }^{7,10}$ Depending on the acoustic pressure amplitude, a cavitation microbubble produced by a $757-\mathrm{kHz}$ sound field should achieve a maximum radius on the order of $1-3 \mu \mathrm{m}$, which is about $\frac{1}{2}$ of the linear resonance radius at that frequency. Ignoring viscosity and thermal conductivity, the $30-\mathrm{MHz}$ backscattering strength for such a bubble is about 140 times larger than that of a typical human erythrocyte (modeled as a fluid sphere). We conclude from this that micrometer-size bubbles are readily detectable. Although ad hoc, this argument supports our con-

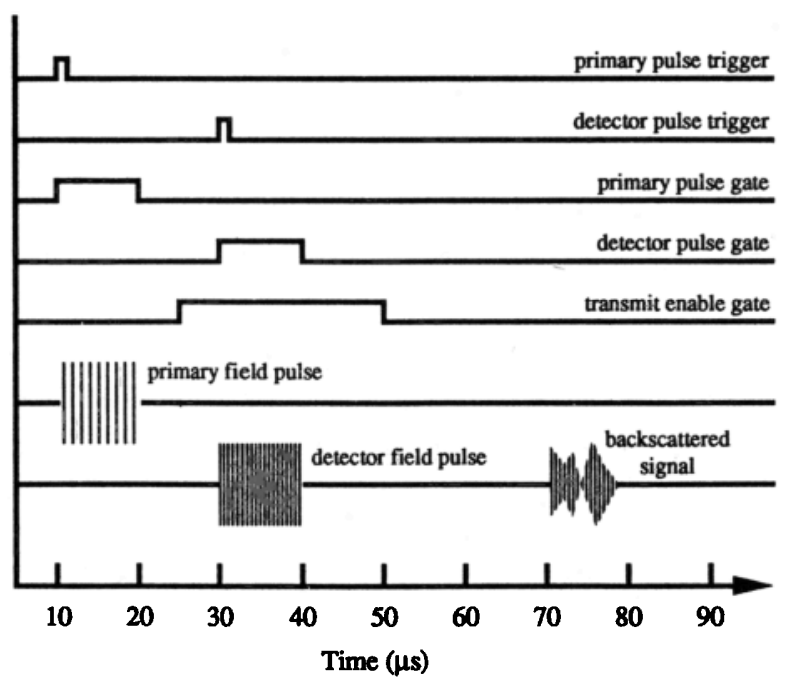

FIG. 6. Timing diagram showing the synchronization of the various triggers and gates employed in the experimental apparatus.

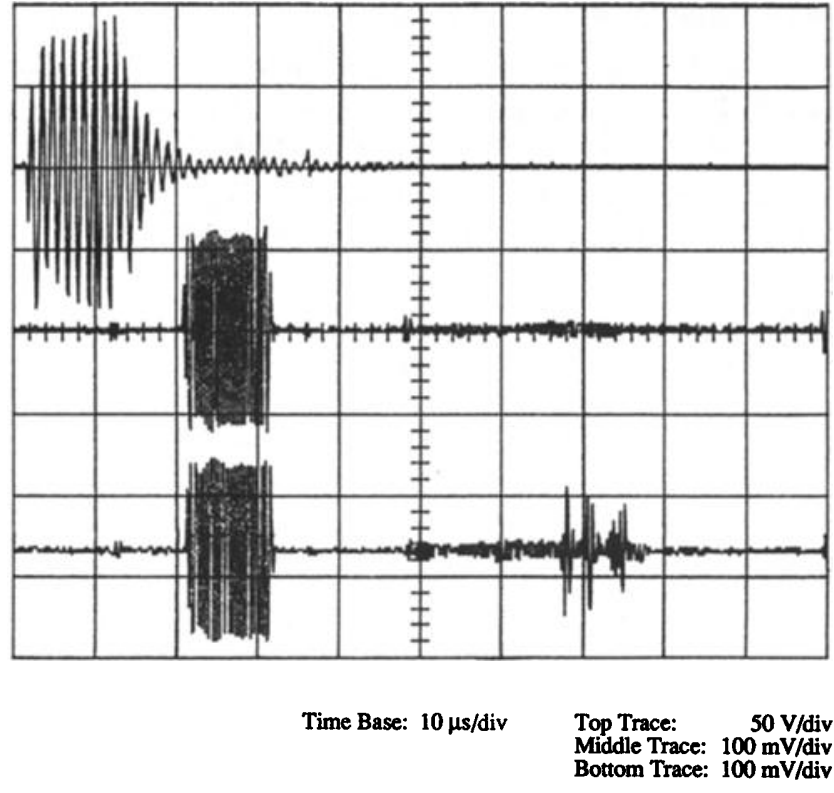

FIG. 7. The 30-MHz ACD output in the absence and presence of cavitation (middle and bottom traces, respectively). The top trace is the electrical tone burst which drives the primary-field transducer. The oscilloscope digitizing rate was $100 \mathrm{Msamples} / \mathrm{s}$.

tention that the ACD is probably capable of detecting transient cavitation activity as minute as the growth and collapse of an individual microbubble. A more rigorous assertion of detectability, which requires independent determination of the ACD output efficiency and receiving sensitivity, will be a topic of future work.

\section{EXPERIMENTAL PROCEDURES}

To evaluate the performance of the $A C D$, we ran a series of experiments in which we suspended controlled quantities of polystyrene microspheres (which served as cavitation nuclei) in distilled, degassed, de-ionized, and filtered water. The mechanisms through which these hydrophobic particles act as nuclei are not well understood. For our purposes, it suffices to say that in the absence of any microspheres, we could stress the water with up to 25 bars of peak negative acoustic pressure (the maximum our system can generate) for as long as $1 \mathrm{~h}$ without observing cavitation activity with either detector, whereas the addition of controlled concentrations of polystyrene spheres resulted in dramatic and reproducible reductions in the measured cavitation thresholds.

To prepare a water sample, we circulated $\approx 15 \ell$ of distilled water through the in-line filters and the test cell for 30 min at atmospheric pressure. We then bypassed the cell and circulated at a reduced pressure for an additional $45 \mathrm{~min}$. During this time, we drained the test cell (which could not withstand a vacuum) while taking precautions to ensure that the water was replaced by filtered air. The cell was then gradually filled with the ultraclean, degassed water and allowed to sit undisturbed for $15 \mathrm{~min}$ during which time preexisting bubbles either dissolved or rose to the surface. Upon suspending the particles (following a procedure described 
below), we allowed the system to sit for an additional $\frac{1}{2} \mathrm{~h}$. After setting the primary-field pulse duration as well as the detector-field pulse amplitude, duration, and delay, we proceeded to measure the transient cavitation thresholds using the procedure described below.

(1) The computer ramped the primary-field pulse amplitude a rate of about $7.8 \mathrm{bars} / \mathrm{min}$. Increasing the ramp rate by a factor of 3 biased the measurements toward higher thresholds (i.e., we ramped past the threshold, an observation also noted by Fowlkes and $\mathrm{Crum}^{6}$ ). Decreasing the rate by a factor of $3 \mathrm{had}$ no effect on the measurements; however, a very slow ramp contributed to operator fatigue. Our choice of ramp rate represents an empirically determined compromise between these competing factors.

(2) While ramping, the operator carefully viewed the detector output (either passive or active) on the oscilloscope screen and depressed a hand-held reset button the instant cavitation activity was observed.

(3) The computer immediately turned off the primary field and recorded the transducer voltage (from which we determined the pressure amplitude) attained just prior to the manual reset command. This value, the transient cavitation threshold, was written to a data file.

(4) The computer delayed $15 \mathrm{~s}$, after which it initiated another measurement by repeating steps (1)-(3).

All experiments were performed at a $1-\mathrm{kHz}$ pulse-repetition frequency with $10-\mu$ s-long primary- and detector-field pulses ( 7 cycles and 300 cycles, respectively). This corresponds to a primary-field duty cycle of $1 \%$, which is typical of diagnostic ultrasound devices. The detector pulse amplitude was fixed at $23 \mathrm{~V}_{\mathrm{pp}}$ and left on regardless of the detection scheme employed. The temperature was $22 \pm 1{ }^{\circ} \mathrm{C}$, and the dissolved gas concentration was varied from $50 \%$ to $90 \%$ of saturation.

Microparticles used in this study came in two monodispersed size ranges. Most of the data were taken with $0.8-\mu \mathrm{m}$ diam noncrosslinked polystyrene microspheres made inhouse by the Yale Chemical Engineering Department. We also used $0.245-\mu \mathrm{m}$-diam carboxyl-stabilized polystyrene microspheres manufactured by Interfacial Dynamics, Inc. ${ }^{11}$ To suspend the particles, we took a highly concentrated sample (4\%-10\% by weight, depending on the sample used) and dispersed it in about $10 \mathrm{~m} \ell$ of degassed, ultraclean water drawn from the cavitation test cell.The test tube containing the diluted mixture was then submerged in an ultrasonic cleaner for $3 \mathrm{~min}$ in order to break up any particle aggregates that might be present. The agitated mixture was then dispersed (by mixing) into the $1.5-\ell$ test cell and allowed to sit undisturbed for at least half an hour. All of the spheres employed in this work are nearly neutrally buoyant (density $=1.05 \mathrm{~g} / \mathrm{m} \ell$ ) and thus remain in suspension for several hours. Concentrations for the final suspensions ranged from $\approx 23 \times 10^{6} \approx 240 \times 10^{6}$ particles $/ \mathrm{m} \ell$.

Experiments consisted of two data runs, each of which typically resulted in 30 measurements. We carried out the runs on separate days using freshly prepared suspensions. Thresholds cited in this article are based on the mean value computed over the entire 60-point sample. Cited precisions are based on either the standard error for the entire sample or the difference in the means of each 30-point data run, whichever is greater.

\section{RESULTS AND DISCUSSION}

\section{A. Cavitation threshold versus dissolved gas concentration}

Figure 8 is a comparison of the thresholds measured using the two detectors for water samples at three different dissolved gas concentrations. These measurements exhibit qualitative agreement with the passively detected results of Atchley et $a .^{6}$ ( $\approx 11$ bars at a $10 \%$ duty cycle) and the chemiluminescence-based measurements performed by Fowlkes and $\mathrm{Crum}^{5}$ ( $\approx 10$ bars for a $10 \%$ duty cycle). The mean thresholds measured with the ACD are less than or equal to those measured passively, although the difference is barely significant and diminishes as the gas concentration increases. Thresholds measured with either detector increase with decreasing gas concentration.

To interpret these results, we must first consider the known ${ }^{5,12}$ sequence of events that most likely leads to the production of a cavitation bubble cloud by pulsed ultrasound. Negative acoustic stress results in the nucleation of a free bubble, which subsequently grows and collapses violently. The bubble breaks up and "seeds" the liquid with several smaller bubbles. If the acoustic pressure amplitude is sufficiently large, these newly created nuclei lead to more cavitation, a cyclic process that eventually results in the formation of a bubble cloud. On the other hand, if the pressure amplitude is too low, the bubbles dissolve and cavitation ceases. Short primary-field pulse durations and long pulse-repetition periods also inhibit bubble cloud formation. This is because there may be insufficient time for a cloud to evolve within a single acoustic pulse, in which case newly formed microbubbles must survive (i.e., keep from dissolving) until the next pulse in order for the cyclic cavitation process to continue.

All experiments were performed using chemically

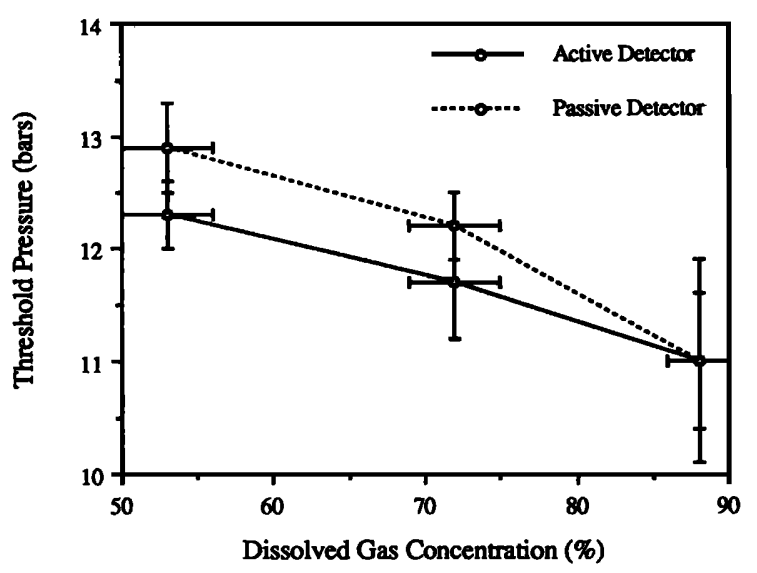

FIG. 8. Transient cavitation threshold versus dissolved gas concentration for a suspension of $0.8-\mu \mathrm{m}$-diam polystyrene spheres in degassed water. The ACD voltage was $23 \mathrm{~V}_{\mathrm{pr}}$ (for all measurements), the particle concentration was $23 \times 10^{6}$ particles $/ \mathrm{m} \ell$, and the temperature was $22 \pm 1{ }^{\circ} \mathrm{C}$. 
clean, de-ionized water. The rate at which a bubble dissolves increases with decreasing bubble size and decreasing dissolved gas concentration. Reducing the gas concentration makes it more difficult for an individual transient cavitation event to cascade into a bubble cloud. Unlike the ACD, the passive cavitation detector may not be sensitive enough to detect the occurrence of a weak cavitation event. If so, passively detected threshold pressures may exceed thresholds measured with the ACD, and the difference should increase with decreasing gas concentration. This observation is consistent with the results presented in Fig. 8.

Dissolved gas concentration also influences the nucleation threshold, which is the minimum acoustic stress required to form a bubble. If this threshold is sufficiently high, bubble nucleation may occur at acoustic pressures which satisfy a priori the requirements for the formation of a bubble cloud. This seems to be the case for the $88 \pm 2 \%$ gas concentration measurements from Fig. 8, where both detectors yielded the same mean threshold pressures. The detectors also show an increasing threshold for detectable cavitation with decreasing gas concentration. This comes as no surprise; even if we do not know the exact nucleation mechanism, it is reasonable to assume that degassing should raise the nucleation threshold. It appears that the ACD provides a measure of the nucleation threshold, whereas the passive detector determines the threshold pressure required for nucleation followed by the formation of a bubble cloud. The extent to which these thresholds differ depends upon the nucleation threshold, the primary-field acoustic parameters (i.e., pulse duration, etc.), and the dissolved gas concentration.

\section{B. Cavitation threshold versus suspended particle concentration}

Figure 9 illustrates the dependence of the cavitation threshold on polystyrene sphere concentration and size for water degassed to $72 \pm 3 \%$ of saturation. Note the dramatic reduction in threshold pressure with increasing particle con-

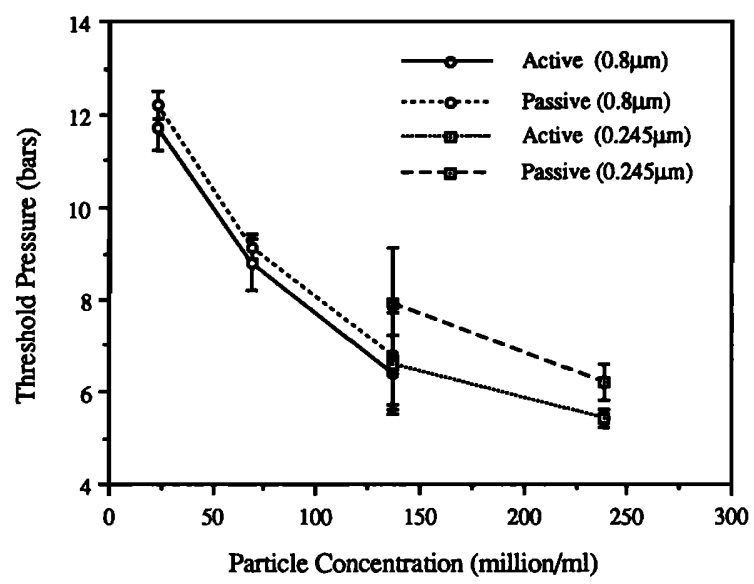

FIG. 9. Transient cavitation threshold versus the concentration of polystyrene spheres suspended in degassed water. The ACD voltage was $23 \mathrm{~V}_{\mathrm{pp}}$ (for all meausurements), the dissolved gas concentration was $72 \pm 3 \%$ of saturation, and the temperature was $22 \pm 1^{\circ} \mathrm{C}$. centration. At this junction we ask ourselves the question: Does this represent a real reduction in the nucleation threshold, or is it merely an experimental bias related to nucleation statistics? It is possible for an imperfect hydrophobic sphere to nucleate a bubble through gas stabilized in a surface crack. Since we employ a finite number of spheres, the probability of finding a gas-ladened sphere in the primary-field focal region at any point in time is less than unity. Indeed, if almost all of the spheres are smooth, this probability could be very small. Depending on the ramp rate, it is possible to ramp through the "true" nucleation threshold pressure, thereby biasing the measurements toward higher values. Increasing the particle concentration should diminish this bias, a result which is consistent with the trend in the data. However, we should also expect a similar reduction with decreasing ramp rate. Aside from inducing operator fatigue, we found that decreasing the ramp rate had little effect on our measurements. The observed threshold reduction is real, and at least one of the mechanisms though which these spheres nucleate microbubbles involves some form of interparticle interaction.

Decreasing the mean particle diameter increased the thresholds measured with the passive detector and had no effect on the ACD measurements. This means that the nucleation threshold is more closely tied to the particle concentration than to the particle size, whereas the pressure required to produce a bubble cloud increases slightly with decreasing particle size. It is conceivable that a suspension of smaller particles might nucleate a smaller bubble, and the seeds produced by the breakup of this bubble could be too small to sustain cavitation unless the acoustic pressure amplitude is increased. This line of reasoning is consistent with the results of Fig. 9.

\section{Cavitation threshold versus detector pulse delay}

By adjusting the detector pulse length and delay, it is possible to control the amount of temporal overlap between the primary- and detector-field pulses. Recall that a $20-\mu \mathrm{s}$ delay corresponds to the simultaneous arrival of the two pulses. Thus, if we insonify a sample with a $10-\mu$ s primary pulse and a $5-\mu$ s detector pulse that has been delayed $25 \mu \mathrm{s}$, we end up interrogating only the second half of the primaryfield tone burst. This capability allows us to determine when cavitation bubbles are produced and how long microbubble remnants persist.

To demonstrate this feature, we generated a 5- $\mu \mathrm{s}$-long detector pulse at a 1-kHz PRF and measured the threshold for bubble production as a function of detector pulse delay. We employed a $137 \times 10^{6}$ particles $/ \mathrm{m} \ell$ suspension of 0.245 $\mu$ m-diam polystyrene spheres in water degassed to $72 \pm 3 \%$ of saturation. These results are shown graphically in Fig. 10, where the width and location of a bar corresponds to the detector pulse duration and delay. The lowest thresholds are represented by the bars labeled (b) and (c), the region in which the primary and detector pulses overlapped. Bar (e) corresponds to bubbles that persisted at least $80 \mu \mathrm{s}$ following the end of the primary pulse, and bar (a) is indicative of bubbles detected prior to the arrival of the primary pulse (i.e., remnants of cavitation produced by the previous 


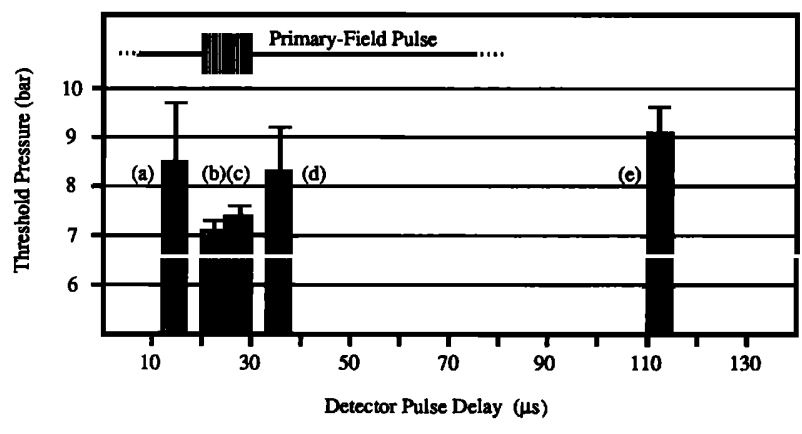

FIG. 10. Transient cavitation threshold versus detector pulse delay for a suspension of $0.8-\mu \mathrm{m}$-diam polystyrene spheres in degassed water. The ACD voltage was $23 \mathrm{~V}_{\mathrm{p}}$ (for all measurements), the dissolved gas concentration was $72 \pm 3 \%$ of saturation, the particle concentration was $137 \times 10^{6}$ particles $/ \mathrm{ml}$, and the temperature was $22 \pm 1{ }^{\circ} \mathrm{C}$. The $10-\mu$ s primary pulse is represented graphically, and each bar corresponds to measurements made with a different delay imposed upon the 5- $\mu$ s detector pulse. For example, the height of bar (b) represents the cavitation threshold measured using a detector pulse which was delayed $20 \mu$ s and, therefore, temporally overlapped the first half of the primary pulse.

pulse). For pressure amplitudes exceeding 8 or 9 bars, microbubbles created by cavitation breakup survive from one primary-field pulse to the next. The difference between the thresholds indicated by bars (a) and (b) represents the difference between the bubble cloud production threshold and the nucleation threshold.

Recall that at $1 \mathrm{MHz}$, the maximum size scales for bubbles produced by acoustic cavitation should be on the order of $1-3 \mu \mathrm{m}$, depending on the acoustic pressure amplitude. It stands to reason that the resulting microbubble remnants should be considerably smaller, say, on the order of $0.1-0.3$ $\mu \mathrm{m}$. By increasing the acoustic pressure amplitude, we can generate larger cavitation bubbles that, in some cases, may result in larger microbubble remnants (owing to the diffusion of gas into the bubble during the growth phase). The approximate time required for a bubble of radius $R$ to completely dissolve in clean water is given by ${ }^{13}$

$$
t_{d}=\frac{P_{0} R^{3}\left(1+2 S / P_{0} R\right)}{6 D S d},
$$

where $P_{0}$ is the ambient pressure, $S$ is the surface tension, $D$ is the diffusion constant, and $d$ is a dimensionless constant dependent upon thermodynamic factors. Corresponding dissolution times for bubbles of radii 0.1 and $0.3 \mu \mathrm{m}$ are on the order of $150 \mu \mathrm{s}$ and $1.4 \mathrm{~ms}$, respectively. Since our pulserepetition period was $1 \mathrm{~ms}$, these calculations suggest that, depending on the acoustic pressure amplitude, microbubble remnants may or may not survive from one primary-field pulse to the next. Increasing the pressure amplitude increases the likelihood that bubbles survive. These observations are consistent with the experimental results given in Fig. 10.

\section{Invasive aspects of active cavitation detection}

The active cavitation detector has one major drawback: It involves the production of acoustic tone bursts and, therefore, could prove to be an invasive manner in which to detect acoustically generated cavitation. Observations made over intervals as long as $45 \mathrm{~min}$ indicate that the $\mathrm{ACD}$ alone will not produce cavitation in our test liquids; the frequency is too high. However, there are important second-order effects left to consider: acoustic streaming and radiation pressure.

When driven by a $23-\mathrm{V}_{\mathrm{pp}}$ pulse at a $1 \%$ duty cycle, the detection transducer produces a steady streaming flow which convects particles through the confocal region at a velocity of $3.6 \pm 0.7 \mathrm{~cm} / \mathrm{s}$. Since this streaming velocity is proportional to the square of the applied voltage and varies linearly with duty cycle, it is easy to manipulate the streaming flow. Using the passive detector, we measured the cavitation threshold as a function of streaming velocity for three different particle suspensions. These results, illustrated in Fig. 11, show a significant reduction in the threshold with increasing velocity and increasing particle concentration. One might argue that by sweeping more particles through the focal region, we are increasing the probability of finding viable cavitation nuclei in the primary-field focus at the proper time. Consider, however, the data points labeled (a) and (b). Although the concentration for point (b) is $\frac{1}{3}$ of that for point (a), its velocity is 6.6 times greater. There are more particles being swept through the focal region per unit time for point (b), yet point (a) has the lower threshold. Like the particle concentration effect, this flow-induced threshold reduction is not a measurement artifact related to nucleation statistics.

At present, we lack a definitive explanation for the cavitation threshold dependence on suspended particle concentration and streaming velocity. In addition to streaming, the ACD can also generate Bjerknes-type interparticle forces that might result in particle agglomeration. This effect would be enhanced by increasing the pressure amplitude and/or the duty cycle (i.e., increase the streaming) or by increasing the particle concentration. A clump of hydrophobic particles may have interstitial spaces that could harbor gas and therefore act as nucleation sites. Although the trends illustrated in Fig. 11 are consistent with this view, we cannot speculate as to the origin of the gas. We can say that, for

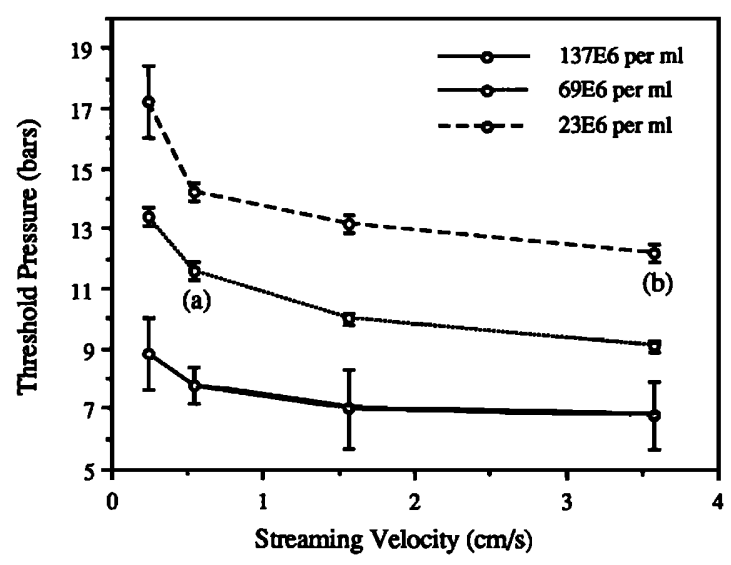

FIG. 11. Transient cavitation threshold versus the steady streaming velocity for three concentrations of $0.8-\mu \mathrm{m}$-diam polystyrene spheres suspended in degassed water. All thresholds were measured with the passive detector. The dissolved gas concentration was $72 \pm 3 \%$ of saturation, and the temperature was $22 \pm 1^{\circ} \mathrm{C}$. 
suspensions of hydrophobic particles, the ACD did alter the nucleation threshold. Although this may not be the case for every experimental situation, one should exercise caution when using the active cavitation detector to study the nucleation characteristics of a fluid.

\section{CONCLUSIONS}

The major contribution of this work is to introduce a new technique for detecting the presence of transient cavitation produced by short pulses of high-frequency ultrasound. Numerous measurements showed that the ACD yielded threshold values that were less than or equal to those measured using a passive detection scheme. The difference may be indicative of a deviation between the nucleation threshold and the threshold for bubble cloud production.

Data obtained with the ACD showed that the nucleation threshold for a suspension of hydrophobic polystyrene spheres decreases with increasing dissolved gas concentration and increasing particle concentration. In addition, increasing the gas content diminished the difference between the nucleation and bubble cloud production thresholds.

The ACD possesses both spatial and temporal resolution. We can therefore determine where and when cavitation activity is taking place. By varying the detector pulse delay, we showed that for large enough primary-field pressure amplitudes, bubble remnants from cavitation activity survived from one primary pulse to the next. Unless the primary pulse is very long, this type of bubble survivability is a necessary condition for bubble cloud production.

Although the thresholds we determined using the ACD ventured as low as 5 bars, all of our measurements were significantly higher than theoretical predictions based on free-bubble models ( $\approx 2.5$ bars). In Ref. 6 , Atchley et al. point out that the growth of a bubble to a detectable size is a two-step process; stress-induced bubble nucleation must be followed by dynamical bubble growth. Free-bubble threshold predictions are applicable only if the nucleation threshold is less than the stress required for bubble growth. Unfortunately, we lack detailed information on how polystyrene spheres nucleate bubbles. Indeed, our experimental results (as well as those in Refs. 6, 8, and 9) suggest that the opposite may be the case. For clean water seeded with polystyrene spheres, the link between measured cavitation thresholds and theoretical predictions (based on free bubbles) is tenuous.

When using the ACD to measure thresholds in polysty- rene sphere suspensions, we found that increasing either the detector pulse amplitude or the duty cycle lowered the nucleation threshold. This invasive characteristic is probably linked to the mechanism by which these spheres nucleate bubbles. Acoustically induced particle agglomeration may be involved, but this is only speculation. The impact of ACD interrogation (or any ultrasonic irradiation for that matter) on microparticle nucleation mechanisms is of fundamental importance and will be the topic of a later report.

\section{ACKNOWLEDGMENTS}

The authors would like to thank Carr Everbach for providing technical advice and assistance, and Christy Holland for calibrating the primary-field transducer, providing technical advice, and offering constructive comments in reviewing the manuscript. This research was funded through NIH Grant 1RO1CA39374.

'R. E. Apfel, “Acoustic cavitation: a possible consequence of biomedical uses of ultrasound," Br. J. Cancer 45 (Suppl. V), 140-146 (1982).

${ }^{2} \mathrm{H}$. G. Flynn, "Generation of transient cavities in liquids by microsecond pulses of ultrasound," J. Acoust. Soc. Am. 72, 1926-1932 (1982).

${ }^{3}$ C. K. Holland and R. E. Apfel, "An improved theory for the prediction of microcavitation thresholds," IEEE Trans. UFFC 36, 204-208 (1989).

${ }^{4}$ A. A. Atchley and L. A. Crum, "Acoustic cavitation and bubble dynamics," in Ultrasound: Its Chemical, Physical, and Biological Effects, edited by K. S. Suslick (VCH, New York, 1988), pp. 1-64.

${ }^{5} \mathrm{~J}$. B. Fowlkes and L. A. Crum, "Cavitation threshold measurements for microsecond length pulses of ultrasound," J. Acoust. Soc. Am. 83, 21902201 (1988)

${ }^{6}$ A. A. Atchley, L. A. Frizzell, R. E. Apfel, C. K. Holland, S. Madanshetty, and R. A. Roy, "Thresholds for cavitation produced in water by pulsed ultrasound," Ultrasonics 26, 280-285 (1988).

${ }^{7}$ R. A. Roy and R. E. Apfel, "Mechanical characterization of microparticles by scattered ultrasound," J. Acoust. Soc. Am. (in press).

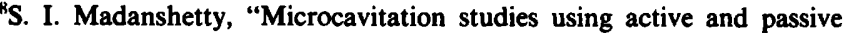
acoustic detection," Ph.D. thesis, Yale University, 1989, available from University Microfilms, Ann Arbor, MI.

${ }^{9} \mathrm{C}$. K. Holland, "Thresholds of transient cavitation produced by pulsed ultrasound in a controlled nuclei environment," Ph.D. thesis, Yale University, 1989, available from University Microfilms, Ann Arbor, MI.

${ }^{10}$ R. A. Roy, "Quantitative particle characterization by scattered ultrasound," Ph.D. thesis, Yale University, 1987, available from University Microfilms, Ann Arbor, MI.

"Interfacial Dynamics Corp., 4814 NE 107th Ave., Suite B, Portland, OR 97220.

${ }^{12}$ H. G. Flynn and C. C. Church, "A mechanism for the generation of cavitation maxima by pulsed ultrasound," J. Acoust. Soc. Am. 76, 505-512 (1984).

${ }^{13}$ P. S. Epstein and M. S. Plesset, "On the stability of gas bubbles in liquidgas solutions," J. Chem. Phys. 18, 1505 (1950). 